\title{
THE DYNAMICS OF METEOROID STREAMS
}

\author{
I. P. WILIAMS \\ Astronomy Unit, Queen Mary and Westfield College, Mile End Rd, London, E1 4NS
}

\begin{abstract}
Meteor showers are seen at regular and frequent intervals on Earth. They are caused by meteoroids (that is small dust grains) in a coherent stream, all moving on similar heliocentric orbits, burning up on encountering the atmosphere of the Earth. Such streams contain $10^{12}$ or more meteoroids, with the mass of the visible meteoroids ranging up to about $1 \mathrm{~g}$. The main evolutionary effect on such streams is gravitational perturbations by the planets. Though graingrain collision may be catastrophic for the two grains involved, it has no effect on the remainder of the stream, other than the fact that there are now two less grains in it. Solar radiation has some effect, but this can be included in the equations of motion. Because of the large numbers of particles involved, meteoroid streams represent a laboratory where many of our dynamical concepts can be tested.

At a basic level, meteoroid streams represent a collective dynamical phenomenon in which all members display roughly the same behavior. One of the fundamental questions which can be investigated is whether the behavior of the mean orbit of the whole stream represents the mean behavior of the stream members. Within the boundaries of some meteor streams lie regions where the orbits are in high order resonance with Jupiter. This also represents a phenomenon of interest. Finally, the possibility exists that some streams are in chaotic regions and it is interesting to investigate whether or not meteoroids in such regions do display chaotic behavior.
\end{abstract}

\section{Introduction}

Rather surprisingly, until the 18 th century very little attention was paid to meteors and meteorites. Indeed, there was a general belief, based mainly on religious grounds, that the universe had to be perfect, and so such debris could not exist. The clearly visible streaks of light in the sky had to be atmospheric in origin. For example, a fall of meteorites near Agen in France on July 24, 1790 was seen by at least 300 people and meteorite fragments were exhibited. However, this did not prevent the editor of the Journal des Sciences Utiles, Pierre Bertholon, from dismissing the whole affair as groundless and physically impossible. On December 14, 1807, a huge fireball was seen over a large section of New England and crashed to Earth near Weston, Connecticut. Sillman, Professor of Chemistry and Kingsley, College Librarian, both of Yale College, collected many samples of this Weston meteorite but nevertheless President Thomas Jefferson is attributed with the, probably apocryphal, remark "it is easier to believe that two Yankee professors would lie than that stones would fall from heaven" . Even before this, however, some had come nearer the truth. Halley (1714) had suggested in 1714 that meteors were formed when "matter formed by some fortuitous concourse of atoms collides with the Earth in its motion about the Sun". Other extra-terrestrial suggestions for the origin of meteors were put foreword by Pringle (1759) and Rittenhouse (1786). In 1794, Chlandi (1794), having systematically analyzed a large amount of published information on meteorite falls, argued for the cosmic origin of fireballs and a generic connection between meteors, fireballs and meteorites. At the turn of the 18th into the 19th century, Benzenberg and Brandes $(1800)$ observed 22 meteors simultaneously from locations a few kilometers apart. They deduced that the mean height was 89 kilometers, a value that is remarkably close to the accepted typical value today. While this 
result does not prove the extra-terrestrial origin of meteors, it nevertheless places them very high in the atmosphere, way above normal atmospheric phenomena.

The study of modern meteor astronomy was probably initiated by the very spectacular Leonid display witnessed in North America on November 13, 1833. From observations of this display, Olmstead (1834) established that the meteors appeared to emerge from a stationary point in the neck of the constellation Leo, that the meteors intersected the atmosphere of the Earth while moving on nearly parallel lines and that the collision velocity was about four miles per second This last conclusion was in fact a significant underestimate. Twining (1834) also reached very similar conclusions but had a slightly higher (but still a considerable underestimate) value of the impact velocity of 14 miles per second. Both men however correctly claimed that this proved the extra-terrestrial origin of meteors. Locke (1834) pointed out that the Perseid meteors also had a fixed point of emergence, or radiant, lying near the star Algol. Herrick (1838) demonstrated the annual nature of the Perseid stream, a fact apparently known to Irish peasants for some time and referred to by them as "the burning tears of St Lawrence". (The festival of St Lawrence is on August 10) (Yeomans, 1991). Newton $(1863,1864,1865)$ noted the comet like orbits of meteor-stream particles. He also pointed out that streams of particles orbiting the Sun on Earth intersecting orbits would collide with the Earth at intervals separated by sidereal rather than tropical years. He further calculated the rate of advancement of the node of the Leonid orbit caused by planetary perturbations. From historical records, he also established a mean time of 33.25 years between major shower events and predicted a spectacular display for the Leonids in 1866. Newton also suggested five possible orbital periods which would agree with this rate of nodal advancement. Adams (1867) showed that, of these five, only the 33.25-year period was consistent with the observed nodal advancement rate. It had thus been established that the orbital period of the Leonid particles was identical to the mean interval between major shower events. In passing, it is interesting to note that this procedure of using the rate of change of the nodal position to accurately determine a period (and hence semi-major axis of the orbit) is the reverse of what is generally done today. In those days, mathematics was more advanced than observational techniques! Le Verrier (1867) assumed a period of 33.25 years for the orbit of the Leonids and calculated the other orbital elements from observations of the Leonid display of 1866 . The similarity of this orbit to that of the newly discovered comet Tempel-Tuttle, 1866 I, was soon obvious. The first actual identification of a comet-meteor stream pair was by Schiaparelli (1867), who showed that the Perseid stream had a similar orbit to that of comet Swift-Tuttle, 1862 III.

The behavior of comet Biela must also have played a significant part in establishing a firm relationship between comets and meteor streams. Inadvertently, in the process, it also set back cometary science by decades. It was argued that meteors are clearly small dust particles, they come from comets, therefore comets must be an agglomeration of dust particles, essentially the sand-bank model for cometary nuclei. In 1826, an Austrian army officer, Captain Wilhelm von Biela, discovered a faint comet (Biela, 1826). The same comet was later independently discovered by Gampart (1826). Clausen (1826) computed an orbit for this comet and recognized that the comets seen in 1772 and 1805 were the same object with a roughly six and 
TABLE I

The major meteor showers.

\begin{tabular}{|c|c|c|c|c|c|}
\hline \multirow{2}{*}{ Name } & \multicolumn{2}{|c|}{ Geometric Radiant } & \multirow{2}{*}{$\begin{array}{l}\text { Date of } \\
\text { Maximum }\end{array}$} & \multirow{2}{*}{$\begin{array}{c}\text { Duration } \\
\text { (days) }\end{array}$} & \multirow{2}{*}{$\begin{array}{l}\text { Maximum } \\
\text { zenith } \\
\text { hour rate }\end{array}$} \\
\hline & R.A. & Dec. & & & \\
\hline Quadrantids & 230 & +49 & January 3 & 4 & 110 \\
\hline Alpha Centaurids & 210 & -59 & February $T$ & 24 & 25 \\
\hline Ly rids & $2 \pi 1$ & +34 & April 22 & 25 & variable \\
\hline Eta Aquarids & 336 & -2 & May 4 & 40 & 50 \\
\hline Perseids & 46 & $+5 T$ & August 12 & $3 \pi$ & 95 \\
\hline Orionids & 95 & +16 & October 22 & 35 & 30 \\
\hline Leonids & 152 & +22 & November 18 & 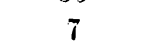 & variable \\
\hline Geminids & 112 & +33 & December 14 & 10 & 110 \\
\hline
\end{tabular}

a half year period. Predictions for the 1832 return turned out to be very accurate and the comet was recovered by Herschel on September 24, 1832. At the next predicted return, in 1839 , observing conditions were not favorable and the comet was not seen. However, based on the previous observations, Santini (1844) predicted a return in 1846 which turned out to be within 10 hours of the correct time. The comet was first recovered in late November 1845 . The first indication that this was to be an abnormal apparition came with the observations of Herrick and Bradley (1846), and, by January 1846, it was clear that a fainter companion also existed 1 or 2 arcseconds North of the main object (Maury, 1846). The two comets travelled together, with tails parallel, for approximately 3 months. The maximum apparent separation achieved was about $25,000 \mathrm{~km}$. At the 1852 return, both comets were again present but fainter, while the separation had increased to about 2 million kilometers. This was to be the last time that comet Biela or its companion were observed. Weiss (1868) calculated that the Earth would intersect the orbit of comet Biela, close to the point where the comet would have been, in 1872 and a strong meteor shower was observed at this time. This phenomenon was repeated in 1885 , 1892 and 1899. It should be noted that low activity had been associated with the Bielid stream in other years. Its radiant lies close to the star Gamma Andromeda and so the shower is also sometimes referred to as the Andromedids.

Thus, before the end of the 19th century, meteors showers had been identified as being caused by a family of small particles, all moving on near identical orbits, burning in the upper atmosphere of the Earth. This would occur at times when the Earth passed through this family on its heliocentric orbit. The source of these particles had also been identified as comets. A list of the major showers observable from Earth are given in table 1, together with some of their major characteristics. The Zenith Hour Rate refers to the number of meteors expected to be seen under ideal conditions should the radiant of the stream be at the Zenith of the observer. The number seen in reality is thus almost always less than this. There followed a period when data collecting continued at a grate rate and the number of identified streams increased significantly. The number of meteor shower-comet 
pairings also increased significantly so that, of the major streams, only the Geminid and Quadrantid streams remained, until recently, apparently without an associated comet.

Further scientific development however required a new understanding of the nature of the cometary nucleus to be developed together with an increased computational capabilities. Hence, the streams could be treated as a true collective phenomenon. An increased mathematical understanding in some cases was also necessary in order to deal with chaos. Each of these developments will be dealt with in turn. This will be followed by some discussion of recent interesting developments in our understanding of some specific streams.

\section{The Formation of a Meteor Stream}

\subsection{Dust Ejection from a Cometary Nucleus}

The breakthrough in terms of our understanding of cometary nuclei and hence the ejection of dust from comets in order to feed meteor streams come principally through the icy conglomerate model proposed by Whipple (1950). The Giotto fly-by of comet Halley in 1986 confirmed the general principles embodied in this model, though of course, many of the point of detail needed modification or amplification (see for example, Whipple, 1987). In this model, a cometary nucleus is a single body composed of a matrix of volatiles, mostly water ice, in which small grains of non-volatiles are embedded in the form of small grains. As a comet approaches perihelion, solar heating causes the volatiles to sublime. This creates an outflow of gas to form the well-known cometary coma. At the same time the embedded grains are released and then accelerated outwards from the nucleus by the drag of the outflowing gas. In the original model, it was envisaged that most of the surface of the nucleus would sublime and be active in releasing grains, but the Giotto results indicate that only a small fraction $f$ of the surface is active at any one time. For comet Halley $f$ is about $15 \%$ (Whipple, 1987). To gain some insight into the dust ejection model, we will give a very simplistic description of the whole process. This will give an order of magnitude estimate of the ejection velocities and grain sizes. The drag on a spherical grain of diameter $d$ moving with relative speed $v$ through a gas of density $\rho$ is given for example by Baines et al. (1965) as

$$
3 F_{D}=\pi d^{2} \rho v W
$$

where $W$ is the mean molecular speed in the gas (roughly the same as the sound speed). When the gas is streaming out with a speed $W$ the grain speed relative to the nucleus is $(W-v)$, so that when the grain is stationary on the nucleus, $v=W$. In theory, the grain could be accelerated until $v=0$, that is until the grain is co-moving with the gas but this is not likely as other forces become important.

For most comets, the gas density, $\rho$, is difficult to measure, but the mass loss rate, $\dot{M}$, may be estimated from ground based observations. Using the equation of continuity,

$$
\dot{M}=4 \pi f \rho W R^{2},
$$


at a distance $R$ from the nucleus, $4 \pi f$ being the solid angle into which gas is ejected. Eliminating $\rho$ from equation 1 gives

$$
F_{D}=\frac{d^{2} v \dot{M}}{12 f R^{2}}
$$

Initially, the gravitational force of the nucleus, $F_{N}$, will be the only force opposing the outward motion of the grain, where

$$
F_{N}=\frac{G M_{N} \pi d^{3} \sigma}{6 R^{2}}
$$

where $\sigma$ is the bulk density of the grains.

Escape will not be possible if on the surface of the nucleus, $F_{N}>F_{D}$, that is if

$$
d>\frac{v \dot{M}}{2 \pi \sigma f G M_{N}} .
$$

For comet Halley, the nucleus mass $M_{N}$ is in the range $5-13 \times 10^{16} \mathrm{~g}$ while a typical mass loss rate $\dot{M}$ was $2 \times 10^{14} \mathrm{~g} \mathrm{y}^{-1}$ (Whipple, 1987). With these values, and assuming a mean molecular weight of 18 for the escaping gas, equation 5 shows that the maximum size of grain that can be carried away from the nucleus by gas drag is about $12 \mathrm{~cm}$. This estimate is in good agreement with the largest size of particles detected in meteoroid showers and is also consistent with the detection of a $1 \mathrm{~g}$ grain by Giotto near the Halley nucleus (McDonnell et al. 1987).

As the grains move out from the nucleus, other forces become important and eventually the motion will be completely determined by the combined effects of solar gravity and solar radiation pressure. The ratio of solar radiation pressure effect to solar gravity is well known (see for example Fox et al. 1982) and is given by

$$
\beta=2.9 \times 10^{-5} /(d \sigma) .
$$

If $\beta$ is greater than one, radiation pressure will clearly drive the grains out of the solar system and such grains will not be present in meteoroid streams. In fact grains with $\beta$ less than unity can escape from the system as their total energy can be positive. Kresak (1974) showed that escape is possible provided

$$
\beta>(1-e) / 2 .
$$

A typical value of $e$ for streams orbits is about 0.9 , and so the minimum grain radius expected to be present in streams is about $5 \times 10^{-3} \mathrm{~cm}$, again very consistent with what is detected.

The second parameter that will influence the formation of meteor streams is the velocity with which a grain leaves the vicinity of the nucleus, that is its speed when it ceases to be influenced by gas drag and becomes a free moving body under heliocentric forces. A rough indication can be obtained by generating an equation 
of motion using the force given in equation 3 and integrating. However, Whipple (1951) has considered this problem in some detail and produced the expression

$$
R=464\left(n \sigma d r^{2.25} R_{C}\right)^{-0.5} \mathrm{cm.s}-1
$$

where $n$ includes both $f$ and an estimate for the amount of energy available for ejection. Here $R_{C}$ is in $\mathrm{km}$ and $r$ in $\mathrm{AU}$. The problem has also been considered recently by Gustafson $(1989 a)$, but the resulting values are similar to those given by the Whipple formula. Insertion of numerical values shows that the escape speed is typically a few hundred to a thousand centimeters per second, and so is very much less than the orbital speed. The ejection is thus but a small perturbation on the heliocentric velocity. Seen in a heliocentric frame, these grains will appear to drift very slowly away from the nucleus while following orbits that are very similar to that of the parent comet.

\subsection{Stream Formation}

If a grain is ejected with a speed relative to the nucleus of $v$ in a direction making an angle $\theta$ with the direction of the orbital velocity of the nucleus, denoted by $V$, then the new energy per unit mass of the grain, $E^{\prime}$, is given by

$$
2 E^{\prime}=V^{2}+v^{2}+2 v V \cos \theta-\frac{G M_{0}(1-\beta)}{r},
$$

while the energy per unit mass of the nucleus $E$ is given by

$$
2 E=-\frac{G M_{0}}{a}=V^{2}-2 \frac{G M_{0}}{r} .
$$

Simple algebra gives

$$
2 E^{\prime}=-\frac{G M_{0}}{a}+v^{2}+2 v V \cos \theta+2 \frac{G M_{0} \beta}{r},
$$

and it can be seen that the energy is positive if $\beta>r / 2 a$ and is the condition used with $r=a(1-e)$ to determine the minimum size of grains remaining in a stream. The specific energy difference between grain and comet is given by

$$
2 \Delta E=2 E^{\prime}-2 E=v^{2}+2 v V \cos \theta+2 \frac{G M_{0} \beta}{r},
$$

while from Kepler's third law we have

$$
\frac{\Delta E}{E}=-\frac{\Delta a}{a}=-\frac{2 \Delta P}{3 P}
$$

As already mentioned, $\Delta E / E$ is generally small and so the changes in $a$ and $P$ will also be small. However, as the period is slightly different grains will arrive back at perihelion at slightly different times from the comet. This delay, or advancement will be repeated every orbit so that, in time, the grains are spread like a doughnut around the cometary orbit. The grains will become spread continuously about the 
orbit after $n$ orbits of the comet, where $n \Delta P=P$. Insertion of numerical values indicates that this spreading typically takes of the order of 100 orbits. This argument is usually used to show that the Leonid stream must thus be young since the grains have clearly not spread into a continuous ring, while the Geminid stream is old since the meteor display is very similar from year to year. While as a general statement the above conclusions must be true, there is an interesting problem concerning the Leonids. Typical numerical values are $V=4 \times 10^{6} \mathrm{~cm} \mathrm{~s}^{-1}, v=10^{4} \mathrm{~cm} \mathrm{~s}^{-1}$ and $a=10.4 \mathrm{AU}$, giving $E=5 \times 10^{11} \mathrm{~cm}^{2} \mathrm{~s}^{-2}$. Thus, even for large grains with $\beta=0$ a typical value for $\Delta E / E$ is 0.08 so that in about 10 orbits, grains should have spread all around the mean orbit. However, Leonid storms have been seen since the 9 th century, that is for 30 orbits and the observational spread, that is the storm duration is only about 2 years in a period of 33.25 years. Of course, all the typical values taken above can be fine tuned, but the nature of the discrepancy is so large that such fine tuning is unlikely to totally resolve the problem. Further details of this point may be found in Williams et al. (1986). Nevertheless, the introduction of the icy conglomerate model for cometary nuclei did allow for significant advances to be made in the study of meteor streams.

\section{Gravitational Perturbations on Streams}

\subsection{Evolution of Meteor Streams}

At a very early stage, the effects of perturbations due to the gravitational field of the planets had been included in discussion. For example, as mentioned earlier, Newton (1863) had included such perturbations in his calculations on the Leonid stream in order to determine the rate of nodal advancement. In early days, means of calculation were extremely tedious, with only mechanical calculators as an aid. The simplest model to use, and the one basically used in these early works, is the one particle model. A single stream particle is taken to represent the stream, and placed at a known point on the orbit. Values for the gravitational forces are then calculated by hand and the resulting change in the motion of the particle computed. The process is then repeated. An advancement on this idea is to average the perturbations over a complete orbit, so that only secular variations remain. Brouwer (1947) generated such a method which worked well even for highly eccentric orbits and this method was used by Whipple and Hamid (1950) to show that 4700 years ago the orbits of the Taurid stream and that of comet Encke were very similar.

Using secular methods, Plavec (1950) showed that the orbit of the Geminid stream is evolving very rapidly, a result confirmed by many other integrations since (for example Hunt et al., 1986, Jones, 1985, Jones and Hawkes, 1986, Gustafson, $1989 \mathrm{~b})$. The variation in the minimum distance from the Geminid stream to Earth and to Venus is shown as Figure 1 . This shows that only 500 years ago, the Geminids were producing a meteor shower on Venus rather than on Earth. The most popular of The secular perturbation methods is Gauss-Halphen-Goryachev method (see for example Hagihara, 1972). This was used by Hagihara (1972) and Babadzhanov and Obrubov $(1980,1983)$ to follow the evolution of meteor streams. Because the averaging reduces considerably the amount of computation that has to be under- 


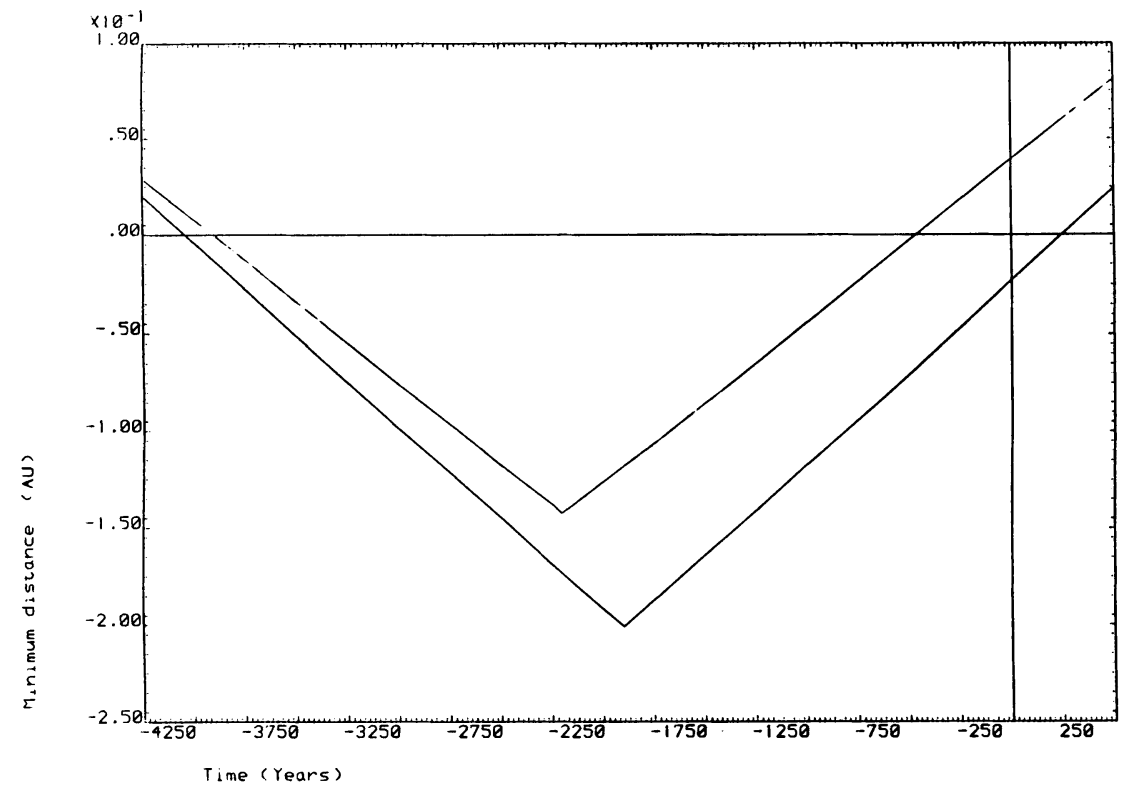

Fig. 1. The minimum distance of the mean Geminid stream from the orbits of the Earth (solid line) and Venus (broken line). The discontinuous change at about 2500 BC: is caused by a switch in the node that is closest.

taken, these method still represent the most efficient way of investigating the long term behavior of the mean meteor stream. Their disadvantage is that through the averaging, peculiar short term behavior of individual particles can be missed.

With the arrival of the electronic computer, it became possible to represent a meteor stream in terms of a number of individual test particles and to numerically integrate the equations of motion of each particle. As well as gravitational effects, it is possible (and indeed this is generally done) to include radiation pressure effects in the equations of motion that are integrated. A brief description of some of the numerical methods used is given in section 3.2. Thus Hamid and Youssef (1963) investigated the evolution of six actual meteors belonging to the Quadrantid stream and concluded that their orbit must have changed drastically over the last millennium. Sherbaum (1970) developed a program of numerical integration based on Cowell's method. This was used by Levin (1972) to show that perturbations by Jupiter on meteor streams produces a general increase in their widths. This highlights the difference between secular perturbation methods, which deals with the evolution of a single orbit into an other single orbit, and the direct approach, where particles initially on the same orbit may be perturbed onto a set of different orbits. Williams et al. (1979) used ten test particles to investigate the long term 


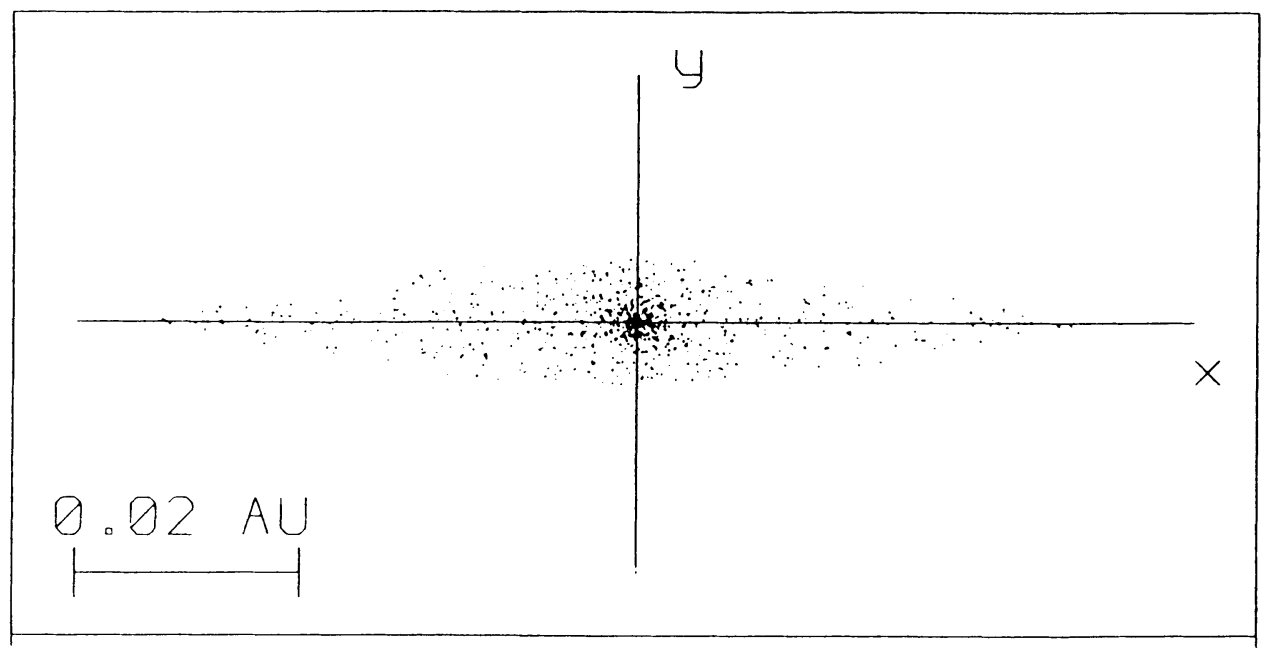

Fig. 2. The cross-section of the Geminid stream at the point closest to the Earth's orbit generated by a model.

evolution of the Quadrantids. The first significant increase in the number of particles used was perhaps by Hughes et al. (1981) when 210 test particles were used to investigate the nodal retrogression rate of the Quadrantid stream. Though the specific term was not used, this investigation also threw up a hint that part of the Quadrantid stream could be chaotic, of which more will be said in section 4 . By 1982, Fox et al. (1982) had increased the number of particles to 1,000 in an investigation of the cross-section obtained for the Geminid meteor stream, assuming the ejection was in a random direction and that the velocity of particles were given by the Whipple(1951) formula The integration interval was only 150 years however. A further dramatic increase in the number of particles used was obtained by Fox et al. (1983), when a more detailed investigation of the cross-section of the Geminids was undertaken, 500,000 particles being used and the evolution time increased to 500 years. This resulted in a moderately realistic stream cross-section being produced and this is shown as Figure 2. By now, the use of direct numerical integration is very widespread, being used for example by Jones and McIntosh (1986) to investigate the relationship between Halley's comet and the $\eta$-Aquarid meteor shower and by Gustafson (1989b) to investigate the relationship between actual Geminid meteors and Asteroid 3200 Phaethon. The orbit of Phaethon is almost identical to that of the Geminid meteor stream (see figure 3) and there seems to be no doubt that they are dynamically related. The "missing" parent comet of the Geminid stream has almost certainly been located. However 3200 Phaethon displays all the characteristics of an asteroid, which leads to the possibility that asteroids can generate meteor streams, as discussed by Hunt et al. (1986), Olsson-Steel (1989) or that Phaethon has been misidentified and that it really is a dormant comet.

This latter notion that many asteroids are dormant comets is under very active 


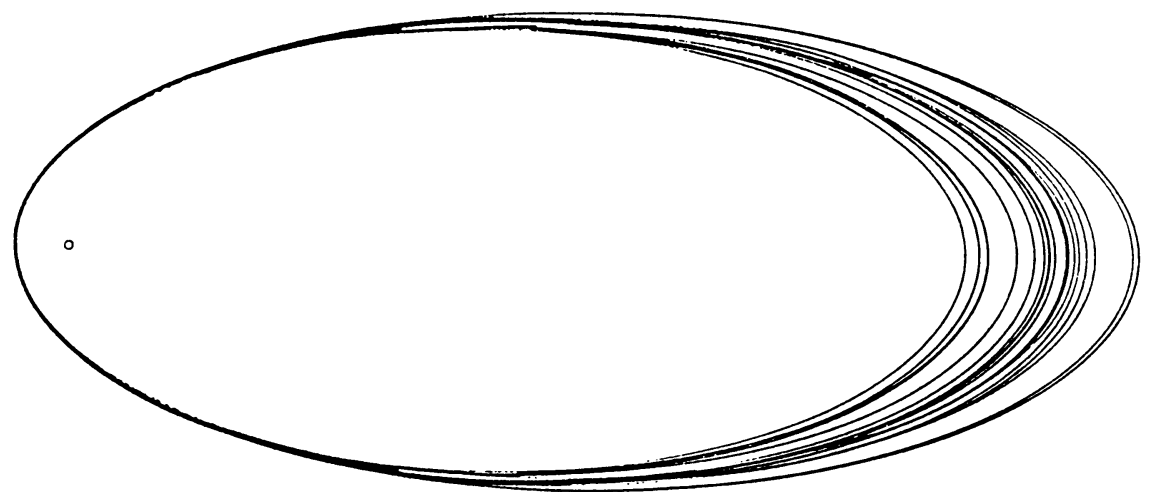

Fig. 3. The orbit of 3200 Phaethon (third orbit from the inside) and the orbits of a set of (ieminid meteoroid orbits, showing the similarity very clearly.

consideration at the present time and is certainly too big an area to be reviewed here. References found in Dahlgren et al. (1991) can be used to study the subject.

Thus, the last 20 years has been one of steady progress in terms of understanding the evolution of meteor streams under the action of gravitational perturbations and it can rightly be claimed that the main effects are now well understood. The essence of this evolution is a change in the orbital parameters of streams on time scales which the modelling indicates is generally matched by observed changes on Earth in either or both of the date of maximum activity and the rate of meteor influx. Some times spectacular events recorded in ancient times can be tied in with present day observed meteor streams by this process. A good example of this is the association by Fox and Williams (1985) of spectacular fireballs in the 11th century with the Monocerotid stream rather than the Geminid stream as might be suspected from the date of the event in December. One development, only recently beginning to influence research, has been the realization, mainly through the work of Lindblad (e.g. 1989) that many of the mean orbits given in the working list of Cook (1973) are based on very few actual meteor orbits. The extreme example is perhaps the $\eta$ Aquarid stream, where the orbit in Cook, and used by many other workers since, is based on only one meteor orbit. A new orbit for this stream is given by Lindblad (1989). Lindblad has also collected together at Lund the catalogue of meteor orbits which may be used to determine new orbits for any stream. Indeed, it is possible to use actual meteors in sufficient numbers, rather than using hypothetical test particles, for the modelling of stream evolution (see for example Wu and Williams in this volume)

\subsection{Numerical Integration Methods}

The integration methods that are currently in common use can be divided into three broad categories, though which side of the division line some of the more 
sophisticated methods actually lies may be a matter of personal preference rather than an absolute definition. The actual name used to describe the category may also vary from person to person These three broad categories are

(i) Taylor series methods

(ii) Single step methods

(iii) Predictor-corrector methods

All the methods reduce the equations of motion to be integrated into a set of first order differential equations in the standard way and solve these equations in sequence. The Taylor series method, as its name implies, assumes that the right hand side of all the differential equations can be differentiated, either analytically or numerically, as often as is required. Taylor series expansions for the variables are then generated so that the numerical value of each variable can then be calculated at a point other than the starting point. The process is repeated as often as is necessary. In principle, this is a very efficient method. In the past, it suffered from the problem that the expressions for the higher derivatives of all the functions involved in the gravitational problem became very long and complex, with the associated difficulty of ensuring mistake-free insertion into the program. This difficulty may be largely overcome now that computer algebra is becoming more widely accessible.

The single step methods, of which the most well known is perhaps the RungeKutta method, uses a fairly simple procedure for evaluating the mean gradient of the function in a given interval and the change in the dependent variable is then obtained by multiplying together the interval length and the mean gradient. The fourth order method is described in most numerical analysis text books and is also generally available as a sub-routine in most computer libraries. For celestial mechanics problems of the form associated with meteor streams a variant such as the Runge-Kutta-Nystrom is generally used. In these variants, two orders of the expansion, are generally produced and the difference between them used to estimate the error involved in the lower order. This allows the step length to be automatically adjusted from step to step. This is of considerable advantage when dealing with very elliptical orbits as the rate of change of the parameters close to perihelion is fast but very slow near aphelion. High order methods have been published by Dormand and Prince (1978) and Dormand et al. (1987).

The third general category of methods is the group of predictor-corrector methods and this can be sub-divided into two main types. Both types require values of the dependent variables being available at a number of different values of the independent variable. In the first type the available data is used in an equation involving a numerical integration in order to predict the next value of the dependent variables in the sequence. With this value a second equation is used in an iterative way to "correct", that is, improve, the accuracy of the predicted value. An example of such a method, found in most text-books, is the Milne-Simpson method (see for example Khabaza, 1969). The other type works in a similar way except that equations based on finite differences are used as predictors and correctors instead of numerical integrations. A typical standard example of this second group is Adams-Bashforth method, while many variants also exist with names hyphenated with Gauss. The main disadvantage of such methods for the solution of problems associated with meteor streams is that a single step method has to be used in order 
to determine a set of starting values and the accuracy of the final solution is then governed in part by the accuracy of the initial single step method.

There also exist many specialized methods, in some cases designed especially with celestial mechanics problems in mind (for example, Brouwer and Clemence, 1961).

\section{Abnormal and Chaotic Behaviour}

In general the evolution of meteor streams, as described by investigations following the methods described above, may be complex but is well-behaved. With minor variations, the evolution of the mean stream computed by using secular perturbation methods agrees very well with the mean of the evolution of individual test particles obtained by numerical integration methods. There may be problems outstanding, but in the main these are problems where the computational model was not sufficiently detailed to follow an observed phenomenon. There does appear to be at least one exception to this however, namely the Quadrantid meteor stream. The first suggestion of peculiar behavior came from the fact that the maximum influx of radio and visible meteors do not coincide in time, implying that the nodal retrogression rate of meteors of different sizes are different (see Hughes et al., 1981). In the same paper, it was shown that the nodal retrogression rate was very sensitive to aphelion distance, this being particularly the case for values close to 5.2 $\mathrm{AU}$, and this behaviour is illustrated in Figure 4. This is not unexpected since at aphelia close to this value, close encounters with Jupiter are possible and, because of the high inclination of the Quadrantid orbit, this is not generally possible if the aphelion distance is much grater. In addition, with a perihelion distance close to 1 $\mathrm{AU}$ and an aphelion distance of order 5.2 $\mathrm{AU}$, the ratio of the Jovian to particle orbital periods are close to a number of ratios of small integer, for example the $2: 1$ ratio exists with an aphelion distance of $5.5 \mathrm{AU}$ and the 5:2 ratio at an aphelion distance of $4.6 \mathrm{AU}$. These are conditions under which very peculiar motion can come about and for which, on investigation of their Lyapunov coefficient, it would not be surprising if it proved to be chaotic. Numerical integrations of the orbits of real Quadrantid orbits have carried out by $\mathrm{Wu}$ and Williams (see this volume) and one such orbit displays peculiar non-periodic jumps in semi-major axis and seems a prime candidate for chaotic behavior. Similar behavior in hypothetical test particles were also found by Gonczi, Rickman and Froeschlé (see this volume), who also claim that the parent of the Quadrantid meteor stream is comet Maccholz. If this proves to be the case, then the parent of all the major streams have now been identified.

\section{Back to the Beginning}

In the introduction, it was pointed out that the Leonid meteor storms and the behavior of comet Biela were significant events in the development of our understanding of meteor streams and their relationship to comets. Rather surprisingly however there could be an other connection between these two events. Bosler and Roure (1937) showed that in 1846 the orbits of the Leonids and comet Biela were 


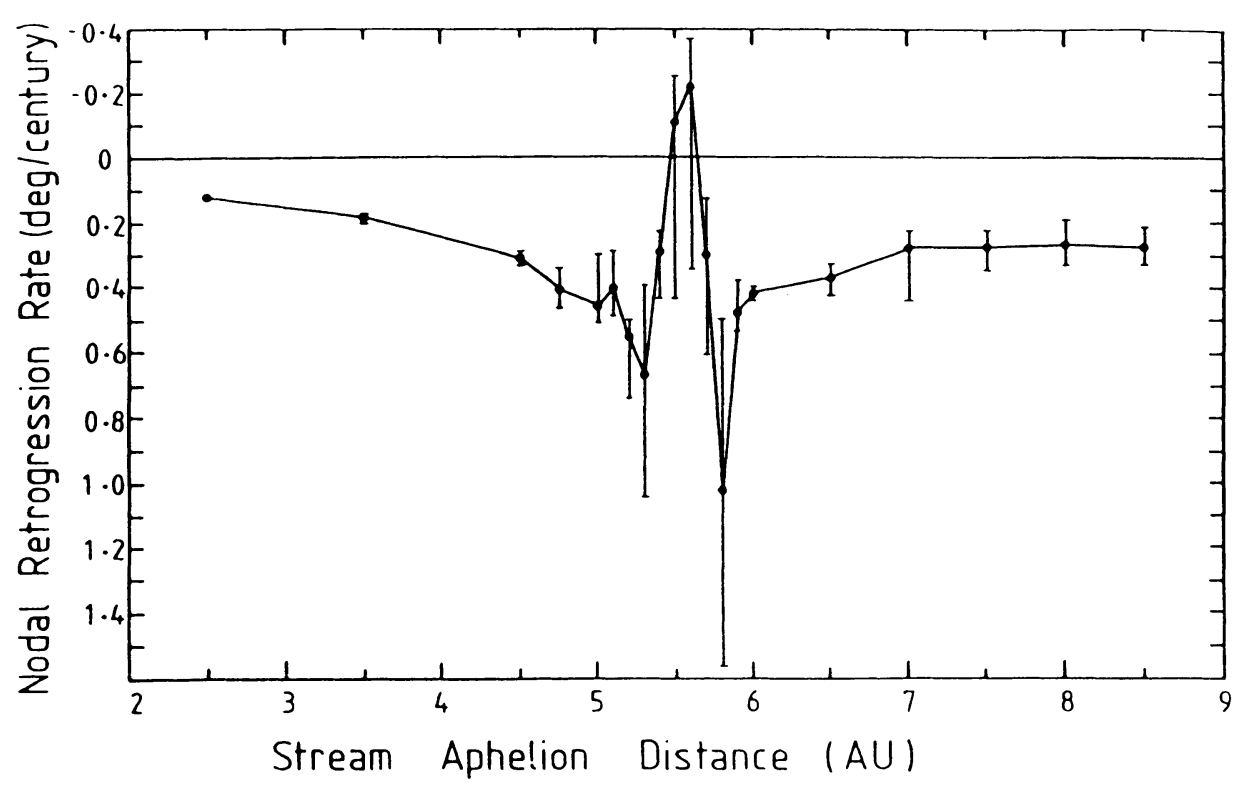

Fig. 4. The variation in the nodal retrogression rate for the Quadrantid stream as a function of aphelion distance

separated by only $0.0265 \mathrm{AU}$ and suggested that it was a collision between the nucleus of Biela and the very dense part of the Leonid stream that caused the Biela nucleus to split into two parts and then disintegrate. They took the published orbital elements for comet Biela for 1846 and also the orbit for the Leonids and used the calculations of Stoney and Downing (1899) to convert this to epoch 1846. They then calculated the shortest distance between these orbits and found it to be as above. Babadzhanov et al. (1991) have reanalyzed the situation, using the current published orbits and the Runge-Kutta-Nystrom numerical integration technique (see Dormand et al., 1987) to follow the orbital evolution. They agree with Bosler and Roure that the orbits of comet Biela and the Leonid stream did indeed only have a separation of $0.026 \mathrm{AU}$ in 1846 ! However, this by itself may be not important .

The reason why we only observe a spectacular meteor storm associated with the Leonid stream (see Williams et al. 1985 for dates) at roughly a 33 year interval is generally agreed to be because meteoroid particles ejected from the parent comet

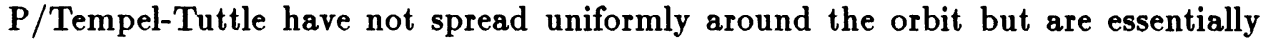
clumped in close proximity of the comet. A spectacular event occurred in 1833 when the particles were close to perihelion and so in 1846 , comet $\mathrm{P} /$ Tempel-Tuttle, and presumably the majority of the meteoroids, were close to aphelion and so nowhere near the supposed intersection point of the two orbits. Since however, the orbits 
do essentially intersect there is a possibility that at some time in the past, both Biela and the Leonid swarm of meteoroids could be at the intersection point at the same time and this possibility was investigated in the above mentioned paper. The closest approach between Biela and the stream was found to be on 1832 Nov 21, when the calculated separation could be as small as $0.002 \mathrm{AU}$ but in reality could be zero due to the uncertainties of the orbit. Finally, the fact that comet Biela may have passed through the densest part of the Leonid meteoroid stream does not in itself imply that this passage was responsible for the breakup of comet Biela, but the possibility is rather intriguing.

\section{References}

Adams, J. C., (1867) Mon. Not. R. ast. Soc. 27, 247

Babadzhanov, P. D. and Obrubov, Y.Y., (1980) in Solid Particles in the Solar System, Eds. Halliday, I. and McIntosh, B.A., D. Reidel Pub. Co., 157.

Babadzhanov, P. D. and Obrubov, Y. Y., (1983) Highlights in Astronomy, vol. 6, Ed. West, R. M., D.Reidel Pub Co., 411.

Babadzhanov, P. D., Zidian Wu, Williams, I. P. and Hughes, D. W., (1991) Mon. Not. R. ast. Soc. (in Press)

Baines, M. J., Williams, I. P. and Asebiomo, A. S., (1965) Mon. Not. R. ast. Soc. $130,63$.

Benzenberg, J. F. and Brandes, H. W., (1800) Annalen der Phys. 6, 224.

Biela, W. von, (1826) Astronomische Nachrichten 4, 443.

Bosler, J. and Roure, H., (1937) Journal des Observateurs 20, 105.

Brouwer, D., (1947), Astron. J. 52, 190.

Brouwer, D. and Clemence, G. M., (1961). Methods of Celestial Mechanics, Academic Press, London.

Chlandi, E. F. F., (1794) Ueber den ursprung der von Pallas gefunden und anderen ahnlichen eisenmassen J. F. Hartnock, Riga.

Clausen, T., (1826) Astronomische Nachrichten 4, 465.

Cook, A. F., (1973) Evolutionary and Physical Properties of Meteoroids, NASA SP-319, p.283.

Dahlgren, M., Fitzsimmons, A., Lagerkvist, C.-I. and Williams, I. P., (1991) Mon. Not. R. astr. Soc. $250,115$.

Dormand, J. R. and Prince, P. J., (1978). Celestial Mechanics 18, 223

Dormand, J. R., El-Mikkai, M. E. A. and Prince, P. J., (1987) J. Numer. Anal. T, 423.

Fox, K. and Williams, I. P., (1985) Mon. Not. R. astr. Soc. $217,407$.

Fox, K., Williams, I. P. and Hughes, D. W., (1982) Mon. Not. R. astr. Soc. $200,313$.

Fox, K., Williams, I. P. and Hughes, D. W., (1983) Mon. Not. R. astr. Soc. $205,1155$.

Froeschlé, Cl. and Scholl, H., (1985) Asteroids Comets Meteors II, Ed Lagerkvist, C.-I., Lindblad, B. A., Lundstedt, H. and Rickman, H. , Uppsala Univ. Reprocentralen.

Gampart, J. F. A., (1826) Astronomische Nachrichten 4, 469.

Gustafson, B. A. S., (1989a) Astrophys. J. 337, 945.

Gustafson, B. A. S., (1989b) Astron. Astrophys. 225, 533.

Hamid, S. E. and Youssef, M. N., (1963) Smithson. Cont. Astrophys. 7, 309.

Hagihara, Y., (1972) Celestial Mechanics vol 2. MIT, Cambridge, USA.

Halley, E., (1714), Phil. Trans. of R. Soc. 29, 159.

Herrick, E. C., (1838) American J. Science 33, 176.

Herrick, E. C. and Bradley F., (1846) American J. of Sci. and Arts 1, 293.

Hughes, D. W., Williams, I. P. and Murray, C. D., (1979) Mon. Not. R. astr. Soc. $189,493$.

Hughes, D. W., Williams, I. P. and Fox, K., (1981) Mon. Not. R. astr. Soc. $195,625$.

Hunt, J., Williams, I. P. and Fox, K., (1985) Mon. Not. R. astr. Soc. $217,533$.

Hunt, J., Fox, K. and Williams, I. P., (1986). Asteroids Comets Meteors II, Ed. Lagerkvist, C.-I., Lindblad, B. A. Lundstedt, H. and Rickman, H., Uppsala Univ. Reprocentralen, 559

Jones, J., (1985). Mon. Not. R. astr. Soc. $217,523$.

Jones, J. and Hawkes, R., (1986) Mon. Not. R. astr. Soc. 223, 479.

Jones, J. and McIntosh, B. A., (1986) Exploration of Comet Halley, ESA SP-250, 233. 
Khabaza, I. M., (1969). Numerical Analysis, Pergamon Press, London.

Kresak, L., (1974) Bull. astr. Inst. Csl. $13,176$.

Le Verrier, U. J. J., (1867) Comptes Rendus 64, 94.

Levin, B. Y., Simonenko, A. N. and Sherbaum, L. M., (1972) in The Motion, Evolution of Orbits and Origin of Comets, Eds. Chebotarev, G. A. et al.., D. Reidel Pub. Co., 454.

Lindblad, B. A., (1989) Asteroids Comets Meteors III, Ed Lagerkvist, C.-I., Rickman, H., Lindblad, B. A. and Lindgren M. , Uppsala Univ. Reprocentralen, 551.

Locke, J., (1834) Cincinati Daily Gazette, Aug. 11 and 12.

McDonnell, J. A. M., Alexander, W. M., Burton, W. M., Bussoleti, E., Evans, G. C., Evans, S. T., Firth, J. G., Grard, R. J. L., Green, S. F., Grun, E., Hanner, M.S., Hughes, D. W., Igenberg, E., Kissel, J., Kuczera, H., Lindblad, B. A., Langevin, Y., Mandeville, J. C., Nappo, S., Pankiewicz, G. S. A., Perry, C. H., Schwehm, G. H., Sekanina, Z., Stevenson, T. J., Turner, R. F., Weishaupt, U., Wallis, M. K.and Zarnecki, J. C., (1987) Astron. Astrophys $187,719$.

Maury, M. F., (1846) Washington Observations 2, 422.

Newton, H. A., (1863) American J. of Sci. and Arts 36, 145.

Newton, H. A., (1864) American J. of Sci. and Arts 37, 377.

Newton, H. A., (1865) American J. of Sci. and Arts 39, 193.

Olmstead, D., (1834) American J. of Science 29, 376

Olsson-Steel, D., (1989) Asteroids Comets Meteors III, Ed Lagerkvist, C.-I., Rickman, H., Lindblad, B. A. and Lindgren M., Uppsala Univ.Reprocentralen, 159

Plavec, M., (1950). Nature 165, 362.

Santini, G., (1844) Astronomische Nachrichten 21171.

Schiaparelli, G. V., (1867) Astronomische Nachrichten 68, 331.

Sherbaum, L. M., (1970) Vestun. Kiev Un-ta. Ser. Astron. 12, 42

Stoney, G. J. and Downing, A. M. W., (1899) Astronomische Nachrichten 149, 33.

Pringle, J., (1759) Phil. Trans. of R. Soc. 51, 259.

Rittenhouse, D., (1786), Trans. American Phil. Soc. 2, 173.

Twining, A. C., (1834) American Jl. Sciences 26, 320.

Weiss, E., (1868) Astronomische Nachrichten 72, 81.

Whipple, F. L., (1950). Astrophys. J. $111,375$.

Whipple, F. L., (1951). Astrophys. J. $113,464$.

Whipple, F. L., (1987). Astron. Astrophys. 187, 852.

Whipple, F. L. and Hamid, S. E. D., (1950), Sky and Telescope 9, 248.

Williams, I. P., Johnson, C. and Fox, K., (1986). Asteroids Comets Meteors II, Eds. Lagerkvist, C.-I., Lindblad, B. A. Lundstedt, H. and Rickman, H., Uppsala Univ. Reprocentralen, 559.

Williams, I. P., Murray, C. D. and Hughes, D. W., (1979) Mon. Not. R. astr. Soc. $189,483$.

Yeomans, D. K., (1991) Comets: a Chronological History of Observations, Science, Myth and Folklore, Wiley and Sons, New York. 